\title{
Infinite Products Involving Binary Digit Sums
}

\author{
Samin Riasat
}

Abstract Let $\left(u_{n}\right)_{n \geq 0}$ denote the Thue-Morse sequence with values \pm 1 . The WoodsRobbins identity below and several of its generalisations are well-known in the literature

$$
\prod_{n=0}^{\infty}\left(\frac{2 n+1}{2 n+2}\right)^{u_{n}}=\frac{1}{\sqrt{2}} .
$$

No other such product involving a rational function in $n$ and the sequence $u_{n}$ seems to be known in closed form. To understand these products in detail we study the function

$$
f(b, c)=\prod_{n=1}^{\infty}\left(\frac{n+b}{n+c}\right)^{u_{n}} .
$$

We prove some analytical properties of $f$. We also obtain some new identities similar to the Woods-Robbins product.

\section{Introduction}

Let $s_{k}(n)$ denote the sum of the digits in the base- $k$ expansion of the non-negative integer $n$. Although we only consider $k=2$, our results can be easily extended to all integers $k \geq 2$. Put $u_{n}=(-1)^{s_{2}(n)}$. In other words, $u_{n}$ is equal to 1 if the binary expansion of $n$ has an even number of 1's, and is equal to -1 otherwise. This is the so-called Thue-Morse sequence with values \pm 1 . We study infinite products of the form

$$
f(b, c):=\prod_{n=1}^{\infty}\left(\frac{n+b}{n+c}\right)^{u_{n}}
$$

Samin Riasat

University of Waterloo, Waterloo, Ontario, Canada, e-mail: sriasateuwaterloo.ca 
The only known non-trivial value of $f$ (up to the relations $f(b, b)=1$ and $f(b, c)=1 / f(c, b))$ seems to be

$$
f\left(\frac{1}{2}, 1\right)=\sqrt{2}
$$

which is the famous Woods-Robbins identity [8, 9]. Several infinite products inspired by this identity were discovered afterwards (see, e.g., [5, 7]), but none of them involve the sequence $u_{n}$. In this paper we compute another value of $f$, namely,

$$
f\left(\frac{1}{4}, \frac{3}{4}\right)=\frac{3}{2}
$$

In Sect.2 we look at properties of the function $f$ and introduce a related function $h$. In Sect. 3 we study the analytical properties of $h$. In Sect. 4 we try to find infinite products of the form $\prod R(n)^{u_{n}}$ admitting a closed form value, with $R$ a rational function.

This paper forms the basis for the paper [3]. While the purpose of [3] is to compute new products of the forms $\prod R(n)^{u_{n}}$ and $\prod R(n)^{t_{n}}, t_{n}$ being the Thue-Morse sequence with values 0,1 , we restrict ourselves in this paper to studying products of the form $\prod R(n)^{u_{n}}$ in greater depth.

\section{General Properties of $f$ and a New Function $h$}

We start with the following result on convergence.

Lemma 1. Let $R \in \mathbb{C}(x)$ be a rational function such that the values $R(n)$ are defined and non-zero for integers $n \geq 1$. Then, the infinite product $\prod_{n} R(n)^{u_{n}}$ converges if and only if the numerator and the denominator of $R$ have same degree and same leading coefficient.

Proof. See [3], Lemma 2.1.

Hence $f(b, c)$ converges for any $b, c \in \mathbb{C} \backslash\{-1,-2,-3, \ldots\}$. Using the definition of $u_{n}$ we see that $f$ satisfies the following properties.

Lemma 2. For any $b, c, d \in \mathbb{C} \backslash\{-1,-2,-3, \ldots\}$,

1. $f(b, b)=1$,

2. $f(b, c) f(c, d)=f(b, d)$,

3. $f(b, c)=\left(\frac{c+1}{b+1}\right) f\left(\frac{b}{2}, \frac{c}{2}\right) f\left(\frac{c+1}{2}, \frac{b+1}{2}\right)$.

Proof. The only non-trivial claim is part 3 To see why it is true, note that $u_{2 n}=u_{n}$ and $u_{2 n+1}=-u_{n}$, so that 


$$
\begin{aligned}
f(b, c) & =\prod_{n=1}^{\infty}\left(\frac{n+b}{n+c}\right)^{u_{n}} \\
& =\left(\frac{1+c}{1+b}\right) \prod_{n=1}^{\infty}\left(\frac{2 n+b}{2 n+c}\right)^{u_{n}} \prod_{n=1}^{\infty}\left(\frac{2 n+1+c}{2 n+1+b}\right)^{u_{n}} \\
& =\left(\frac{1+c}{1+b}\right) \prod_{n=1}^{\infty}\left(\frac{n+\frac{b}{2}}{n+\frac{c}{2}}\right)^{u_{n}} \prod_{n=1}^{\infty}\left(\frac{n+\frac{c+1}{2}}{n+\frac{b+1}{2}}\right)^{u_{n}} \\
& =\left(\frac{c+1}{b+1}\right) f\left(\frac{b}{2}, \frac{c}{2}\right) f\left(\frac{c+1}{2}, \frac{b+1}{2}\right)
\end{aligned}
$$

as desired.

One can ask the natural question: is $f$ the unique function satisfying these properties? What if we impose some continuity/analyticity conditions?

Using the first two parts of Lemma 2 we get

$$
f(b, c) f(d, e)=\frac{f(b, c) f(c, d) f(d, e) f(d, c)}{f(c, d) f(d, c)}=\frac{f(b, e) f(d, c)}{f(c, c)}=f(b, e) f(d, c) .
$$

Hence the third part may be re-written as

$$
f(b, c)=\frac{f\left(\frac{b}{2}, \frac{b+1}{2}\right)}{b+1} / \frac{f\left(\frac{c}{2}, \frac{c+1}{2}\right)}{c+1} .
$$

This motivates the following definition.

Definition 1. Define the function

$$
h(x):=f\left(\frac{x}{2}, \frac{x+1}{2}\right) .
$$

Then Eqs. (1) and (2) give the following result.

Lemma 3. For any $b, c \in \mathbb{C} \backslash\{-1,-2,-3, \ldots\}$,

$$
f(b, c)=\frac{c+1}{b+1} \cdot \frac{h(b)}{h(c)} .
$$

So understanding $f$ is equivalent to understanding $h$, in the sense that each function can be completely evaluated in terms of the other. Moreover, taking $c=b+\frac{1}{2}$ in Eq. (3) and then using Eq. (2) gives the following result.

Lemma 4. The function $h$ defined by Eq. (2) satisfies the functional equation

$$
h(x)=\frac{x+1}{x+\frac{3}{2}} h\left(x+\frac{1}{2}\right) h(2 x) .
$$


Again one may ask: is $h$ the unique solution to Eq. (4)? What about monotonic/continuous/smooth solutions?

An approximate plot of $h$ is given in Fig. 1 with the infinite product truncated at $n=100$.

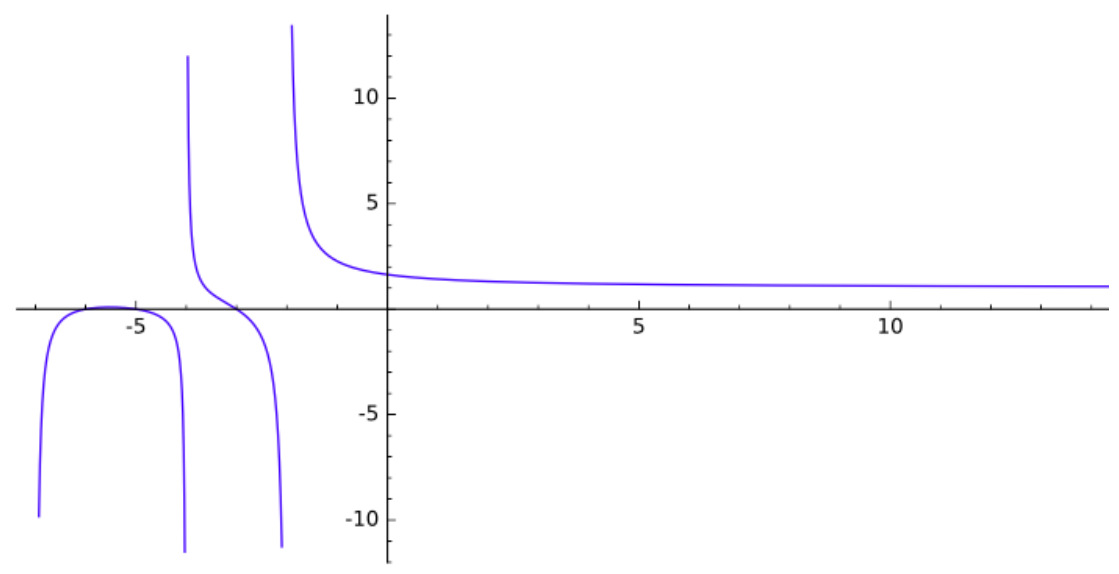

Fig. 1 Approximate plot of $h(x)$

\section{Analytical Properties of $h$}

The following lemma forms the basis for the results in this section.

Lemma 5. For $b, c \in(-1, \infty)$,

1. if $b=c$, then $f(b, c)=1$.

2. if $b>c$, then

$$
\left(\frac{c+1}{b+1}\right)^{2}<f(b, c)<1 \text {. }
$$

3. if $b<c$, then

$$
1<f(b, c)<\left(\frac{c+1}{b+1}\right)^{2} .
$$

Proof. Using Lemma2 2 it suffices to prove the second statement.

Let $b>c>-1$ and put

$$
a_{n}=\log \left(\frac{n+b}{n+c}\right), \quad S_{N}=\sum_{n=1}^{N} a_{n} u_{n}, \quad U_{N}=\sum_{n=1}^{N} u_{n} .
$$


Note that $a_{n}$ is positive and strictly decreasing to 0 . Using $s_{2}(2 n)+s_{2}(2 n+1) \equiv 1$ $(\bmod 2)$ it follows that $U_{n} \in\{-2,-1,0\}$ and $U_{n} \equiv n(\bmod 2)$ for each $n$. Using summation by parts,

$$
S_{N}=a_{N+1} U_{N}+\sum_{n=1}^{N} U_{n}\left(a_{n}-a_{n+1}\right) .
$$

So $-2 a_{1}<S_{N}<0$ for large $N$. Exponentiating and taking $N \rightarrow \infty$ gives the desired result.

Lemmas 3 immediately imply the following results.

Theorem 1. $h(x) /(x+1)$ is strictly decreasing on $(-1, \infty)$ and $h(x)(x+1)$ is strictly increasing on $(-1, \infty)$.

Theorem 2. For $b, c \in(-1, \infty), f(b, c)$ is strictly decreasing in $b$ and strictly increasing in $c$.

Theorem 3. For $x \in(-2, \infty)$,

$$
1<h(x)<\left(\frac{x+3}{x+2}\right)^{2} .
$$

We now give some results on differentiability.

Theorem 4. $h(x)$ is smooth on $(-2, \infty)$.

Proof. Recall the definition of $h$ :

$$
h(x)=\prod_{n=1}^{\infty}\left(\frac{2 n+x}{2 n+1+x}\right)^{u_{n}} .
$$

Then taking $b=x / 2$ and $c=(x+1) / 2$ in Eqs. (5) shows that the sequence $S_{n}$ of smooth functions on $(-2, \infty)$ converges pointwise to $\log h$.

Differentiating with respect to $x$ gives

$$
S_{N}^{\prime}=\sum_{n=1}^{N} \frac{u_{n}}{(2 n+x)(2 n+1+x)}=\sum_{n=1}^{N} u_{n}\left(\frac{1}{2 n+x}-\frac{1}{2 n+1+x}\right) .
$$

Hence 


$$
\begin{aligned}
\left|S_{N}^{\prime}-S_{M}^{\prime}\right| & \leq \sum_{n=M+1}^{N}\left(\frac{1}{2 n+x}-\frac{1}{2 n+1+x}\right) \\
& \leq \sum_{n=M+1}^{N}\left(\frac{1}{2 n-1+x}-\frac{1}{2 n+1+x}\right) \\
& =\frac{1}{2 M+1+x}-\frac{1}{2 N+1+x} \\
& <\frac{1}{2 M-1} \rightarrow 0
\end{aligned}
$$

as $M \rightarrow \infty$, for any $x \in(-2, \infty)$ and $N>M$. Thus $S_{n}^{\prime}$ converges uniformly on $(-2, \infty)$, which shows that $\log h$, hence $h$, is differentiable on $(-2, \infty)$.

Now suppose that derivatives of $h$ up to order $k$ exist for some $k \geq 1$. Note that

$$
S_{N}^{(k+1)}=(-1)^{k} k ! \sum_{n=1}^{N} u_{n}\left(\frac{1}{(2 n+x)^{k+1}}-\frac{1}{(2 n+1+x)^{k+1}}\right) .
$$

As before,

$$
\begin{aligned}
\left|S_{N}^{(k+1)}-S_{M}^{(k+1)}\right| & \leq k ! \sum_{n=M+1}^{N}\left(\frac{1}{(2 n+x)^{k+1}}-\frac{1}{(2 n+1+x)^{k+1}}\right) \\
& \leq k ! \sum_{n=M+1}^{N}\left(\frac{1}{(2 n-1+x)^{k+1}}-\frac{1}{(2 n+1+x)^{k+1}}\right) \\
& =\frac{k !}{(2 M+1+x)^{k+1}}-\frac{k !}{(2 N+1+x)^{k+1}} \\
& <\frac{k !}{(2 M-1)^{k+1}} \rightarrow 0
\end{aligned}
$$

as $M \rightarrow \infty$, for any $x \in(-2, \infty)$ and $N>M$. Hence $S_{n}^{(k+1)}$ converges uniformly on $(-2, \infty)$, i.e., $h^{(k)}$ is differentiable on $(-2, \infty)$.

Therefore, by induction, $h$ has derivatives of all orders on $(-2, \infty)$.

Theorem 5. Let $a \geq 0$. Then

$$
\log h(x)=\log h(a)+\sum_{k=1}^{\infty} \frac{(-1)^{k-1}}{k}\left(\sum_{n=2}^{\infty} \frac{u_{n}}{(n+a)^{k}}\right)(x-a)^{k}
$$

for $x \in[a-1, a+1]$.

Proof. Let $H(x)=\log h(x)$. By Theorem 4

$$
H^{(k+1)}(x)=(-1)^{k} k ! \sum_{n=2}^{\infty} \frac{u_{n}}{(n+x)^{k+1}} .
$$

Hence 


$$
\left|H^{(k+1)}(x)\right| \leq k ! \sum_{n=2}^{\infty} \frac{1}{|n+x|^{k+1}} \leq k ! \sum_{n=2}^{\infty} \frac{1}{(n+a-1)^{k+1}}
$$

for $x \in[a-1, a+1]$. So by Taylor's inequality, the remainder for the Taylor polynomial for $H(x)$ of degree $k$ is absolutely bounded above by

$$
\frac{1}{k+1}\left(\sum_{n=2}^{\infty} \frac{1}{(n+a-1)^{k+1}}\right)|x-a|^{k+1}
$$

which tends to 0 as $k \rightarrow \infty$, since $a \geq 0$ and $|x-a| \leq 1$. Therefore $H(x)$ equals its Taylor expansion about $a$ for $x$ in the given range.

\section{Infinite Products}

Recall that

$$
f(b, c)=\prod_{n=1}^{\infty}\left(\frac{n+b}{n+c}\right)^{u_{n}} .
$$

From Lemma2 we see that

$$
\prod_{n=1}^{\infty}\left(\frac{(n+b)\left(n+\frac{b+1}{2}\right)\left(n+\frac{c}{2}\right)}{(n+c)\left(n+\frac{c+1}{2}\right)\left(n+\frac{b}{2}\right)}\right)^{u_{n}}=\frac{c+1}{b+1}
$$

for any $b, c \neq-1,-2,-3, \ldots$, and if $b, c \neq 0,-1,-2, \ldots$, then

$$
\prod_{n=0}^{\infty}\left(\frac{(n+b)\left(n+\frac{b+1}{2}\right)\left(n+\frac{c}{2}\right)}{(n+c)\left(n+\frac{c+1}{2}\right)\left(n+\frac{b}{2}\right)}\right)^{u_{n}}=1 .
$$

Some interesting identities can be obtained from Eqs. (6)-(7). For example, in Eq. [6, taking $c=(b+1) / 2$ gives

$$
\prod_{n=1}^{\infty}\left(\frac{(n+b)\left(n+\frac{b+1}{4}\right)}{\left(n+\frac{b+3}{4}\right)\left(n+\frac{b}{2}\right)}\right)^{u_{n}}=\frac{b+3}{2(b+1)}
$$

while taking $b=0$ gives

$$
\prod_{n=1}^{\infty}\left(\frac{\left(n+\frac{1}{2}\right)\left(n+\frac{c}{2}\right)}{(n+c)\left(n+\frac{c+1}{2}\right)}\right)^{u_{n}}=c+1
$$

for any $b, c \neq-1,-2,-3, \ldots$

We now turn our attention to the functional equation Eq. (4). Recall that it reads

$$
h(x)=\frac{x+1}{x+\frac{3}{2}} h\left(x+\frac{1}{2}\right) h(2 x) .
$$


Taking $x=0$ gives

$$
h(0)=\frac{2}{3} h\left(\frac{1}{2}\right) h(0) .
$$

Since $1<h(0)<9 / 4$ by Theorem 3, cancelling $h(0)$ from both sides gives $h(1 / 2)=$ $3 / 2$. This shows that

$$
\prod_{n=0}^{\infty}\left(\frac{4 n+3}{4 n+1}\right)^{u_{n}}=2 \text {. }
$$

Next, taking $x=1 / 2$ in Eq. (4) gives

$$
h\left(\frac{1}{2}\right)=\frac{3}{4} h(1)^{2}
$$

hence $h(1)=\sqrt{2}$ (since $1<h(1)<16 / 9$ by Theorem 3 and we recover the WoodsRobbins product

$$
\prod_{n=0}^{\infty}\left(\frac{2 n+2}{2 n+1}\right)^{u_{n}}=\sqrt{2}
$$

Similarly, taking $x=-1 / 2$ in Eq. (4) gives

$$
h\left(-\frac{1}{2}\right)=\frac{1}{2} h(0) h(-1)=\frac{1}{2} f\left(0, \frac{1}{2}\right) f\left(-\frac{1}{2}, 0\right)=\frac{1}{2} f\left(-\frac{1}{2}, \frac{1}{2}\right),
$$

i.e.,

$$
\prod_{n=1}^{\infty}\left(\frac{(4 n-1)(2 n+1)}{(4 n+1)(2 n-1)}\right)^{u_{n}}=\frac{1}{2}
$$

Taking $x=1$ in Eq. (4) gives

$$
h(1)=\frac{4}{5} h\left(\frac{3}{2}\right) h(2)
$$

hence $h(3 / 2) h(2)=5 \sqrt{2} / 4$ and this gives

$$
\prod_{n=0}^{\infty}\left(\frac{(4 n+3)(2 n+2)}{(4 n+5)(2 n+3)}\right)^{u_{n}}=\frac{1}{\sqrt{2}} .
$$

Taking $x=3 / 2$ in Eq. (4) and using the previous result gives

$$
h(2)^{2} h(3)=\frac{3}{\sqrt{2}}
$$

which is equivalent to

$$
\prod_{n=0}^{\infty}\left(\frac{(2 n+2)(n+1)}{(2 n+3)(n+2)}\right)^{u_{n}}=\frac{1}{\sqrt{2}}
$$


Eqs. 10 -14 can also be combined in pairs to obtain other identities.

\section{Concluding Remarks}

The quantity $h(0) \approx 1.62816$ appears to be of interest [1, 4]. It is not known whether its value is irrational or transcendental. We give the following explanation as to why $h(0)$ might behave specially in a sense.

Note that the only way non-trivial cancellation occurs in the functional equation Eq. (4) is when $b=0$. Likewise, non-trivial cancellation occurs in Eq. (1) or property 3 in Lemma2 only for $(b, c)=(0,1 / 2)$ and $(1 / 2,0)$. That is, the victim of any such cancellation is always $h(0)$ or $h(0)^{-1}$. So one must look for other ways to understand $h(0)$.

Using the only two known values $h(1 / 2)=3 / 2$ and $h(1)=\sqrt{2}$, the following expressions for $h(0)$ can be obtained from Theorem 5

- By taking $x=0$ and $a=1$,

$$
h(0)=\sqrt{2} \exp \left(-\sum_{k=1}^{\infty} \frac{1}{k} \sum_{n=2}^{\infty} \frac{u_{n}}{(n+1)^{k}}\right) .
$$

- By taking $x=1$ and $a=0$,

$$
h(0)=\sqrt{2} \exp \left(\sum_{k=1}^{\infty} \frac{(-1)^{k}}{k} \sum_{n=2}^{\infty} \frac{u_{n}}{n^{k}}\right) .
$$

- By taking $x=0$ and $a=1 / 2$,

$$
h(0)=\frac{3}{2} \exp \left(\sum_{k=1}^{\infty} \frac{1}{k} \sum_{n=2}^{\infty} \frac{u_{2 n+1}}{(2 n+1)^{k}}\right) .
$$

- By taking $x=1 / 2$ and $a=0$,

$$
h(0)=\frac{3}{2} \exp \left(\sum_{k=1}^{\infty} \frac{(-1)^{k}}{k} \sum_{n=2}^{\infty} \frac{u_{2 n}}{(2 n)^{k}}\right) .
$$

The Dirichlet series

$$
\sum_{n=0}^{\infty} \frac{u_{n}}{(n+1)^{k}} \quad \text { and } \quad \sum_{n=1}^{\infty} \frac{u_{n}}{n^{k}}
$$

appearing in the above expressions were studied by Allouche and Cohen [2].

Acknowledgements This work is part of a larger joint work [3] with Professors Jean-Paul Allouche and Jeffrey Shallit. I thank the professors for helpful discussions and comments. I also thank the anonymous referees for their feedback. 


\section{References}

1. J.-P. Allouche, Thue, Combinatorics on words, and conjectures inspired by the Thue-Morse sequence, J. de Théorie des Nombres de Bordeaux, 27, no. 2 (2015), 375-388.

2. J.-P. Allouche, H. Cohen, Dirichlet series and curious infinite products, Bull. London Math. Soc. 17 (1985), 531-538.

3. J.-P. Allouche, S. Riasat, J. Shallit, More infinite products: Thue-Morse and the Gamma function, Ramanujan J. (2018). https: / /doi.org/10.1007/s11139-017-9981-7

4. J.-P. Allouche, J. Shallit, The ubiquitous Prouhet-Thue-Morse sequence. Sequences and their Applications: Proceedings of SETA '98, (1999), 1-16.

5. J.-P. Allouche, J. Shallit, Infinite products associated with counting blocks in binary strings, J. London Math. Soc. 39 (1989), 193-204.

6. J.-P. Allouche, J. Shallit, Automatic Sequences. Theory, Applications, Generalizations, Cambridge University Press, Cambridge, 2003.

7. J.-P. Allouche, J. Sondow, Infinite products with strongly B-multiplicative exponents, Ann. Univ. Sci. Budapest. Sect. Comput. 28 (2008), 35-53. [Errata: Ann. Univ. Sci. Budapest. Sect. Comput. 32 (2010), 253.]

8. D. Robbins, Solution to problem E 2692, Amer. Math. Monthly 86 (1979), 394-395.

9. D. R. Woods, Elementary problem proposal E 2692, Amer. Math. Monthly 85 (1978), 48. 